\title{
Almost periodic solution of a discrete Nicholson's blowflies model with delay and feedback control
}

Xiaoying Chen ${ }^{1 *}$, Chunling Shi ${ }^{1}$ and Yiqin Wang ${ }^{2}$

"Correspondence:

snailkitty@126.com

'Department of Computer

Engineering, Fuzhou University

Zhicheng College, Fuzhou, Fujian

350002, P.R. China

Full list of author information is

available at the end of the article

\begin{abstract}
In this paper, we propose a discrete Nicholson's blowflies model with feedback control. Sufficient conditions are obtained for the permanence. By means of an almost periodic functional hull theory, we show that the almost periodic system has a uniqueness of globally attractive almost periodic solution. Moreover, a suitable example and its numerical simulation are given to illustrate the feasibility of the main results.
\end{abstract}

MSC: 34D20; 34K14; 39A24

Keywords: discrete; Nicholson's blowflies model; almost periodic solution; feedback control

\section{Introduction}

In this paper, we propose and study the permanence and global attractivity of an almost positive solution of the following discrete Nicholson's blowflies model with delay and feedback control:

$$
\begin{aligned}
& \Delta x(n)=-\alpha(n) x(n)+\beta(n) x\left(n-\tau_{1}\right) e^{-\gamma(n) x\left(n-\tau_{1}\right)}-c(n) x(n) u(n), \\
& \Delta u(n)=-a(n) u(n)+b(n) x\left(n-\tau_{2}\right),
\end{aligned}
$$

where $x(n)$ is the density of the species at time $n$ and $u(n)$ is the control variable at time $n$. For any bounded sequence $\{\eta(n)\}$, we define $\eta^{+}=\sup _{n \in Z}\{\eta(n)\}$ and $\eta^{-}=\inf _{n \in Z}\{\eta(n)\}$.

Throughout this work, we use the following assumptions:

$\left(H_{1}\right)\{\alpha(n)\},\{\beta(n)\},\{\beta(n)\},\{a(n)\},\{b(n)\}$, and $\{c(n)\}$ are bounded nonnegative almost periodic sequences such that

$$
\begin{aligned}
& 0<\alpha^{-} \leq \alpha(n) \leq \alpha^{+}<1, \quad 0<\beta^{-} \leq \beta(n) \leq \beta^{+}, \quad 0<\gamma^{-} \leq \gamma(n) \leq \gamma^{+}, \\
& 0<a^{-} \leq a(n) \leq a^{+}<1, \quad 0<b^{-} \leq b(n) \leq b^{+}, \quad 0<c^{-} \leq c(n) \leq c^{+}, \quad n \in Z .
\end{aligned}
$$
(http://creativecommons.org/licenses/by/4.0/), which permits unrestricted use, distribution, and reproduction in any medium, provided you give appropriate credit to the original author(s) and the source, provide a link to the Creative Commons license, and indicate if changes were made. 
We consider system (1.1) with the following initial conditions:

$$
\begin{aligned}
& x(\theta)=\varphi(\theta) \geq 0, \quad \theta \in\{-\tau,-\tau+1, \ldots,-2,-1,0\}, \\
& \varphi(0)>0, \quad u(0)>0, \quad \tau=\max \left\{\tau_{1}, \tau_{2}\right\} .
\end{aligned}
$$

It is not difficult to see that solutions of (1.1) with the initial condition (1.2) are well defined and remain positive for all $n \geq 0$.

As we know, Nicholson's blowflies model belongs to a class of biological systems and its analog equation has attracted more attention because of its extensively realistic significance. Topics such as existence, uniqueness, and exponential convergence of almost periodic solutions of the system were extensively investigated, and many excellent results have been derived; see [1-4] and the references cited therein. However, in the study of ecology, a discrete model is more significant in practice than a differential model as these species are short in life and have non-overlapping generations. Discrete-time models can also provide efficient computational models of continuous models for numerical simulation. Therefore, lots have been done on discrete-time population models. To mention a few cases, we refer the reader to [5-16]. For example, Zhang et al. [5] have studied the dynamic behavior of the following autonomous discrete differential equation:

$$
\Delta x(n)=-\alpha x(n)+\beta x(n-\tau) e^{-\gamma x(n-\tau)}
$$

Recently, some scholars [6-8] paid attention to the non-autonomous discrete Nicholson's blowflies models. They mainly studied the existence and exponential convergence of positive almost periodic solutions of the models.

On the other hand, ecosystems in the real world are continuously disturbed by unpredictable forces which can result in changes in the biological parameters such as survival rates. Of practical interest in ecology is the question of whether or not an ecosystem can withstand those unpredictable disturbances which persist for a finite period of time. In the language of control variables, we call the disturbance functions control variables. In 1992, Gopalsamy and Weng [17] introduced a feedback control variable into logistic models and discussed the asymptotic behavior of solutions in logistic models with feedback controls, in which the control variables satisfy a certain differential equation. We also refer to [9, $10,15,16,18-24]$ for a further study of population models with feedback control. Zhao and Wang [23] discussed a general Nicholson's blowflies model with feedback control as follows:

$$
\begin{aligned}
& \frac{d x_{1}}{d t}=-\alpha(t) x_{1}(t)+\beta(t) x_{1}\left(t-\tau_{1}(t)\right) e^{-\gamma(t) x\left(t-\tau_{2}(t)\right)}-c(t) x_{1}(t) x_{2}(t-\eta(t)), \\
& \frac{d x_{2}}{d t}=-\lambda(t) x_{2}(t)+b(t) x_{1}(t-\delta(t)) .
\end{aligned}
$$

However, to the best of the authors' knowledge, to this day, studies of the general discrete Nicholson's blowflies model with feedback control are fairly rare. This is the main motivation of this paper.

For the population models with feedback controls, as we well know, an important subject is to study the effects of the feedback controls on the dynamical behavior of the models. Thus, an important and interesting open problem is proposed here, that is, whether or not 
in system (1.1) the feedback control influences the permanence of the species. In [6-8], the main approach to investigating the existence and stability of the almost periodic solutions of the system is using the fixed point theorem and the Lyapunov functional method. Is there any more convenient method to solve a similar problem as regards system (1.1)?

The rest of the paper is organized as follows. In the next section, we give some definitions and present some useful lemmas. In Section 3, some sufficient conditions for the permanence of system (1.1) are obtained. Then, in Section 4, we establish a sufficient condition for the existence and uniqueness of a globally attractive almost periodic solution. Finally, the main result is illustrated by giving an example with its numerical simulation.

\section{Preliminaries}

Lemma 2.1 [24] Assume that $A>0$ and $y(0)>0$, and further suppose that

(1)

$$
y(n+1) \leq A y(n)+B(n), \quad n=1,2, \ldots .
$$

Then for any integer $k \leq n$,

$$
y(n) \leq A^{k} y(n-k)+\sum_{i=0}^{k-1} A^{i} B(n-i-1) .
$$

Especially, if $A<1$ and $B$ is bounded above with respect to $M$, then

$$
\limsup _{n \rightarrow \infty} y(n) \leq \frac{M}{1-A}
$$

(2)

$$
y(n+1) \geq A y(n)+B(n), \quad n=1,2, \ldots
$$

Then for any integer $k \leq n$,

$$
y(n) \geq A^{k} y(n-k)+\sum_{i=0}^{k-1} A^{i} B(n-i-1) .
$$

Especially, if $A<1$ and $B$ is bounded below with respect to $m$, then

$$
\limsup _{n \rightarrow \infty} y(n) \geq \frac{m}{1-A} .
$$

Definition 2.2 System (1.1) is said to be permanent if there exist positive constants $M_{1}$, $M_{2}, m_{1}, m_{2}$, which are independent of the solutions of the system, such that any positive solution $(x(n), u(n))$ of system (1.1) satisfies

$$
\begin{aligned}
& m_{1} \leq \liminf _{n \rightarrow \infty} x(n) \leq \limsup _{n \rightarrow \infty} x(n) \leq M_{1}, \\
& m_{2} \leq \liminf _{n \rightarrow \infty} u(n) \leq \limsup _{n \rightarrow \infty} u(n) \leq M_{2} .
\end{aligned}
$$


Definition 2.3 [25] A sequence $x: Z \rightarrow R$ is called an almost periodic sequence if the $\varepsilon$-translation set of $x$

$$
E\{\varepsilon, x\}=\tau \in Z:|x(n+\tau)-x(n)|<\varepsilon, \quad \forall n \in Z,
$$

is a relatively dense set in $Z$ for all $\varepsilon>0$; that is, for any given $\varepsilon>0$, there exists an integer $l(\varepsilon)>0$ such that each interval of length $l(\varepsilon)$ contains an integer $\tau \in E\{\varepsilon, x\}$ with

$$
|x(n+\tau)-x(n)|<\varepsilon, \quad \forall n \in Z
$$

The integer $\tau$ is called an $\varepsilon$-translation number of $x(n)$.

Definition 2.4 [26] Let $D$ be an open subset of $R^{m}$. A function $f: Z \times D \rightarrow R^{m}$ is said to be almost periodic in $n$ uniformly for $x \in D$, if for any $\varepsilon>0$ and any compact set $S \subset D$, there exists a positive integer $l=l(\varepsilon, S)$ such that any interval of length $l$ contains an integer $\tau$, for which

$$
|f(n+\tau, x)-f(n, x)|<\varepsilon, \quad \forall(n, x) \in Z \times S .
$$

$\tau$ is called an $\varepsilon$-translation number of $f(n, x)$.

Definition 2.5 [27] The hull of $f$, denoted by $H(f)$, is defined by

$$
H(f)=\left\{g(n, x): \lim _{k \rightarrow \infty} f\left(n+\tau_{k}, x\right)=g(n, x) \text { uniformly on } Z \times S\right\}
$$

for some sequence $\left\{\tau_{k}\right\}$, where $S$ is any compact set in $D$.

Lemma 2.6 [28] $\{x(n)\}$ is an almost periodic sequence if and only iffor any integer sequence $\left\{k_{i}^{\prime}\right\}$, there exists a subsequence $\left\{k_{i}\right\} \subset\left\{k_{i}^{\prime}\right\}$ such that the sequence $\left\{x\left(n+k_{i}\right)\right\}$ converges uniformly for all $n \in Z$ as $i \rightarrow \infty$. Furthermore, the limit sequence is also an almost periodic sequence.

\section{Permanence}

Set

$$
\begin{aligned}
& M_{1}=\frac{\beta^{+}}{\alpha^{-} \gamma^{-} e}, \quad M_{2}=\frac{b^{+} M_{1}}{a^{-}}, \\
& m_{1}=\min \left\{\frac{1}{\gamma^{+}} \ln \frac{\beta^{-}}{\alpha^{+}+c^{+} M_{2}}, \frac{\beta^{-}}{\alpha^{+}+c^{+} M_{2}} M_{1} e^{-\gamma^{+} M_{1}}\right\}, \quad m_{2}=\frac{b^{-} m_{1}}{a^{+}} .
\end{aligned}
$$

Theorem 3.1 Assume that $\left(H_{1}\right)$ holds; assume further that

$\left(H_{2}\right) \alpha^{+}+c^{+} M_{2}<\min \left\{1, \beta^{-}\right\}$

holds, then system (1.1) is permanent. 
Proof Let $(x(n), u(n))$ be any positive solution of system (1.1); from the first equation of (1.1), it follows that

$$
\begin{aligned}
x(n+1) & \leq(1-\alpha(n)) x(n)+\beta(n) x\left(n-\tau_{1}\right) e^{-\gamma(n) x\left(n-\tau_{1}\right)} \\
& \leq\left(1-\alpha^{-}\right) x(n)+\beta^{+} x\left(n-\tau_{1}\right) e^{-\gamma^{-} x\left(n-\tau_{1}\right)} .
\end{aligned}
$$

This combined with the fact that $\sup _{u \geq 0} u e^{-\gamma u}=\frac{1}{\gamma e}$ leads to

$$
x(n+1) \leq\left(1-\alpha^{-}\right) x(n)+\frac{\beta^{+}}{\gamma^{-} e} .
$$

By applying Lemma 2.1 to (3.2), it follows that

$$
\limsup _{n \rightarrow \infty} x(n) \leq \frac{\beta^{+}}{\alpha^{-} \gamma^{-} e} \stackrel{\text { def }}{=} M_{1} .
$$

For any positive constant $\varepsilon$ small enough, it follows from (3.3) that there exists large enough $N_{1}$ such that

$$
x(n) \leq M_{1}+\varepsilon, \quad \text { for all } n \geq N_{1} .
$$

Equation (3.4) combined with the second equation of (1.1) leads to

$$
u(n+1) \leq\left(1-a^{-}\right) u(n)+b^{+}\left(M_{1}+\varepsilon\right), \quad \text { for all } n \geq N_{1}+\tau .
$$

By applying Lemma 2.1 , for any positive solution $(x(n), u(n))$, it follows from $(3.5)$ that

$$
\limsup _{n \rightarrow \infty} u(n) \leq \frac{b^{+}\left(M_{1}+\varepsilon\right)}{a^{-}} .
$$

Setting $\varepsilon \rightarrow 0$ in the above inequality leads to

$$
\limsup _{n \rightarrow \infty} u(n) \leq \frac{b^{+} M_{1}}{a^{-}} \stackrel{\text { def }}{=} M_{2} .
$$

For any positive constant $\varepsilon$ small enough, without loss of generality, from $\left(H_{2}\right)$ we may assume that $\alpha^{+}+c^{+}\left(M_{2}+\varepsilon\right)<\min \left\{1, \beta^{-}\right\}$. It follows from (3.3) and (3.6) that there exists a large enough $N_{2}>N_{1}+\tau$ such that

$$
x(n) \leq M_{1}+\varepsilon, \quad u(n) \leq M_{2}+\varepsilon, \quad \text { for all } n \geq N_{2} .
$$

Now we prove that any positive solution $x(n)$ of system (1.1) and (1.2) satisfies

$$
\liminf _{n \rightarrow \infty} x(n)>0 .
$$

Suppose, for the sake of contradiction, $\liminf _{n \rightarrow \infty} x(n)=0$.

We define

$$
t(n)=\max \left\{s: s \leq n, x(s)=\min _{0 \leq \xi \leq n} x(\xi)\right\} .
$$


Observe that $t(n) \rightarrow \infty$ as $n \rightarrow \infty$ and

$$
\lim _{n \rightarrow \infty} x(t(n))=0
$$

However, $x(t(n))=\min _{0 \leq \xi \leq n} x(\xi)$, and so $x(t(n+1))-x(t(n)) \leq 0$, which implies that

$$
\begin{aligned}
0 \geq & x(t(n+1))-x(t(n))=-\alpha(t(n)) x(t(n)) \\
& +\beta(t(n)) x(t(n)-\tau) e^{-\gamma(t(n)) x(t(n)-\tau)}-c(t(n)) x(t(n)) u(t(n)) \\
\geq & -\alpha^{+} x(t(n))+\beta^{-} x(t(n)-\tau) e^{-\gamma^{+} x(t(n)-\tau)} \\
& -c^{+}\left(M_{2}+\varepsilon\right) x(t(n)), \quad \text { for all } n \geq \max \left\{t(n), N_{2}\right\},
\end{aligned}
$$

and therefore

$$
0=\lim _{n \rightarrow \infty}\left(\alpha^{+}+c^{+}\left(M_{2}+\varepsilon\right)\right) x(t(n)) \geq \lim _{n \rightarrow \infty} \beta^{-} x(t(n)-\tau) e^{-\gamma^{+} x(t(n)-\tau)}
$$

Hence,

$$
\lim _{n \rightarrow \infty} x(t(n)-\tau)=0
$$

Thus, together with (3.10) and the definition of $t(n)$, we have

$$
\alpha^{+}+c^{+}\left(M_{2}+\varepsilon\right) \geq \liminf _{n \rightarrow \infty} \frac{\beta^{-} x(t(n)-\tau)}{x(t(n))} e^{-\gamma^{+} x(t(n)-\tau)} \geq \liminf _{n \rightarrow \infty} \beta^{-} e^{-\gamma^{+} x(t(n)-\tau)}=\beta^{-},
$$

which contradicts with $\alpha^{+}+c^{+}\left(M_{2}+\varepsilon\right)<\beta^{-}$. Hence (3.8) holds.

We next prove that there exists a positive constant $m_{1}$ such that $\liminf _{n \rightarrow \infty} x(n) \geq m_{1}$.

Define

$$
\eta=\liminf _{n \rightarrow \infty} x(n)
$$

and

$$
h=\min \{g(\eta), g(M)\}, \quad \text { where } g(x)=x e^{-\gamma^{+} x} .
$$

This combined with system (1.1) and (3.8) leads to

$$
x(n+1) \geq\left(1-\alpha^{+}\right) x(n)+\beta^{-} h-c^{+}\left(M_{2}+\varepsilon\right) x(n), \quad \text { for all } n \geq N_{2} \text {. }
$$

Thus, together with $0<\alpha^{+}+c^{+}\left(M_{2}+\varepsilon\right)<1$, we have

$$
\begin{aligned}
\eta & =\liminf _{n \rightarrow \infty} x(n) \\
& \geq \liminf _{n \rightarrow \infty}\left[\left(x(0)-\frac{\beta^{-} h}{\alpha^{+}+c^{+}\left(M_{2}+\varepsilon\right)}\right)\left(1-\alpha^{+}-c^{+}\left(M_{2}+\varepsilon\right)\right)^{n}+\frac{\beta^{-} h}{\alpha^{+}+c^{+}\left(M_{2}+\varepsilon\right)}\right] \\
& =\frac{\beta^{-} h}{\alpha^{+}+c^{+}\left(M_{2}+\varepsilon\right)} .
\end{aligned}
$$


Setting $\varepsilon \rightarrow 0$ in the above inequality, we have

$$
\eta=\liminf _{n \rightarrow \infty} x(n) \geq \frac{\beta^{-} h}{\alpha^{+}+c^{+} M_{2}} .
$$

If $h=g(\eta)$, then $\eta \geq \frac{\beta^{-}}{\alpha^{+}+c^{+} M_{2}} \eta e^{-\gamma^{+} \eta}$. So we have $\eta \geq \frac{1}{\gamma^{+}} \ln \frac{\beta^{-}}{\alpha^{+}+c^{+} M_{2}}$.

If $h=g\left(M_{1}\right)$, it follows from (3.11) that $\eta \geq \frac{\beta^{-}}{\alpha^{+}+c^{+} M_{2}} M_{1} e^{-\alpha^{+} M_{1}}$.

The above inequality leads to

$$
\liminf _{n \rightarrow \infty} x(n) \geq \min \left\{\frac{1}{\gamma^{+}} \ln \frac{\beta^{-}}{\alpha^{+}+c^{+} M_{2}}, \frac{\beta^{-}}{\alpha^{+}+c^{+} M_{2}} M_{1} e^{-\alpha^{+} M_{1}}\right\} \stackrel{\text { def }}{=} m_{1} .
$$

For any positive constant $\varepsilon$ small enough, without loss of generality, assume that $\varepsilon<\frac{1}{2} m_{1}$, from (3.12) we know that there exists a large enough $N_{3}>N_{2}$ such that

$$
x(n) \geq m_{1}-\varepsilon, \quad \text { for all } n \geq N_{3} .
$$

Equation (3.13) combined with the second equation of (1.1) leads to

$$
u(n+1) \geq\left(1-a^{+}\right) u(n)+b^{-}\left(m_{1}-\varepsilon\right), \quad \text { for all } n \geq N_{3}+\tau .
$$

By applying Lemma 2.1, for any positive solution $(x(n), u(n))$, it follows from (3.14) that

$$
\limsup _{n \rightarrow \infty} u(n) \geq \frac{b^{-}\left(m_{1}-\varepsilon\right)}{a^{+}} .
$$

Setting $\varepsilon \rightarrow 0$ in the above inequality leads to

$$
\limsup _{n \rightarrow \infty} u(n) \geq \frac{b^{-} m_{1}}{a^{+}} \stackrel{\text { def }}{=} m_{2}
$$

Equations (3.3), (3.6), (3.12), and (3.15) show that if the assumptions $\left(H_{1}\right)$ and $\left(H_{2}\right)$ hold, system (1.1) is permanent. This ends the proof of Theorem 3.1.

\section{Global attractivity of almost periodic solution}

The main purpose of this paper is to investigate the existence and uniqueness of globally attractive almost periodic solution of system (1.1).

First of all, we investigate the attractivity of the solution of (1.1).

Theorem 4.1 Assume that $\left(H_{1}\right)$ and $\left(H_{2}\right)$ hold; suppose further that

$\left(H_{3}\right) \gamma^{-} m_{1}>1$

and

$\left(H_{4}\right) \alpha^{-}+c^{-} m_{2}>b^{+}+\frac{1}{e^{2}} \beta^{+}, a^{-}>c^{+} M_{1}$,

hold, then system (1.1) is globally attractive. That is, for any positive solutions $(x(n), u(n))$ and $(p(n), q(n))$ of system (1.1), we have $\lim _{n \rightarrow \infty}(x(n)-p(n))=0, \lim _{n \rightarrow \infty}(u(n)-q(n))=0$. 
Proof For any solutions $(x(n), u(n))$ and $(p(n), q(n))$ of system (1.1), it follows from Theorem 3.1 that

$$
\begin{array}{ll}
m_{1} \leq \liminf _{n \rightarrow \infty} x(n) \leq \limsup _{n \rightarrow \infty} x(n) \leq M_{1}, & m_{1} \leq \liminf _{n \rightarrow \infty} p(n) \leq \limsup _{n \rightarrow \infty} p(n) \leq M_{1}, \\
m_{2} \leq \liminf _{n \rightarrow \infty} u(n) \leq \limsup _{n \rightarrow \infty} u(n) \leq M_{2}, & m_{2} \leq \liminf _{n \rightarrow \infty} q(n) \leq \limsup _{n \rightarrow \infty} q(n) \leq M_{2} .
\end{array}
$$

For any positive constant $\varepsilon>0$ small enough, there exists an integer $n_{0}$ such that, for all $n \geq n_{0}$,

$$
\begin{array}{ll}
m_{1}-\varepsilon \leq x(n), & p(n) \leq M_{1}+\varepsilon, \\
m_{2}-\varepsilon \leq u(n), & q(n) \leq M_{2}+\varepsilon .
\end{array}
$$

Using the mean value theorem, we get

$$
x(n) e^{-x(n)}-p(n) e^{-p(n)}=(1-\theta(n)) e^{-\theta(n)}(x(n)-p(n)),
$$

where $\theta(n)$ lies between $x(n)$ and $p(n)$.

Let

$$
V_{1}(n)=|x(n)-p(n)|+|u(n)-q(n)|
$$

Then, from system (1.1) and (4.2), we get

$$
\begin{aligned}
\Delta V_{1}(n)= & V_{1}(n+1)-V_{1}(n) \\
\leq & -\alpha(n)|x(n)-p(n)| \\
& +\beta(n)\left|x\left(n-\tau_{1}\right) e^{-\gamma(n) x\left(n-\tau_{1}\right)}-p\left(n-\tau_{1}\right) e^{-\gamma(n) p\left(n-\tau_{1}\right)}\right| \\
& -c(n) u(n)|x(n)-p(n)|+c(n) p(n)|u(n)-q(n)| \\
& -a(n)|u(n)-q(n)|+b(n)\left|x\left(n-\tau_{2}\right)-p\left(n-\tau_{2}\right)\right| \\
\leq & -\alpha(n)|x(n)-p(n)| \\
& +\beta(n)\left|\left(1-\theta\left(n-\tau_{1}\right)\right) e^{-\theta\left(n-\tau_{1}\right)}\left(x\left(n-\tau_{1}\right)-p\left(n-\tau_{1}\right)\right)\right| \\
& -c(n) u(n)|x(n)-p(n)|+c(n) p(n)|u(n)-q(n)| \\
& -a(n)|u(n)-q(n)|+b(n)\left|x\left(n-\tau_{2}\right)-p\left(n-\tau_{2}\right)\right| .
\end{aligned}
$$

According to $\left(H_{3}\right),(4.3)$, and the fact that $\max _{x \in[1,+\infty]}(1-x) e^{-x}=\frac{1}{e^{2}}$, for $n \geq n_{0}+\tau$, we have

$$
\begin{aligned}
\Delta V_{1}(n) \leq & -\alpha(n)|x(n)-p(n)| \\
& +\frac{1}{e^{2}} \beta(n)\left|x\left(n-\tau_{1}\right)-p\left(n-\tau_{1}\right)\right| \\
& -c(n) u(n)|x(n)-p(n)|+c(n) p(n)|u(n)-q(n)| \\
& -a(n)|u(n)-q(n)|+b(n)\left|x\left(n-\tau_{2}\right)-p\left(n-\tau_{2}\right)\right| .
\end{aligned}
$$


Let

$$
\begin{aligned}
& V_{2}(n)=\sum_{u=n-\tau_{1}}^{n-1} \frac{1}{e^{2}} \beta\left(u+\tau_{1}\right)|x(u)-p(u)|, \\
& V_{3}(n)=\sum_{u=n-\tau_{2}}^{n-1} b\left(u+\tau_{2}\right)|x(u)-p(u)| .
\end{aligned}
$$

Then

$$
\begin{aligned}
& \Delta V_{2}(n)=\frac{1}{e^{2}} \beta\left(n+\tau_{1}\right)|x(n)-p(n)|-\frac{1}{e^{2}} \beta(n)\left|x\left(n-\tau_{1}\right)-p\left(n-\tau_{1}\right)\right|, \\
& \Delta V_{3}(n)=b\left(n+\tau_{2}\right)|x(n)-p(n)|-b(n)\left|x\left(n-\tau_{2}\right)-p\left(n-\tau_{2}\right)\right| .
\end{aligned}
$$

Define

$$
V(n)=V_{1}(n)+V_{2}(n)+V_{3}(n) .
$$

Then it follows from (4.4) and (4.5) that

$$
\begin{aligned}
\Delta V(n) \leq & \left(-\alpha(n)+\frac{1}{e^{2}} \beta\left(n+\tau_{1}\right)-c(n) u(n)+b\left(n+\tau_{2}\right)\right)|x(n)-p(n)| \\
& +(c(n) p(n)-a(n))|u(n)-q(n)| .
\end{aligned}
$$

From (4.1) and (4.6), for $n>n_{0}+\tau$, we obtain

$$
\begin{aligned}
\Delta V(n) \leq & \left(-\alpha^{-}+\frac{1}{e^{2}} \beta^{+}-c^{-}\left(m_{2}-\varepsilon\right)+b^{+}\right)|x(n)-p(n)| \\
& +\left(c^{+}\left(M_{1}+\varepsilon\right)-a^{-}\right)|u(n)-q(n)| .
\end{aligned}
$$

From condition $\left(H_{4}\right)$ and the above $\varepsilon$, we can choose $\delta$ small enough such that

$$
\delta=\min \left\{\alpha^{-}+c^{-}\left(m_{2}-\varepsilon\right)-b^{+}-\frac{1}{e^{2}} \beta^{+}, a^{-}-c^{+}\left(M_{1}+\varepsilon\right)\right\}>0 .
$$

From (4.7) and (4.8), we obtain

$$
\Delta V(n) \leq-\delta(|x(n)-p(n)|+|u(n)-q(n)|) .
$$

Summing both sides of the above inequalities from $n_{0}+\tau$ to $n$, we have

$$
\sum_{s=n_{0}+\tau}^{n}(V(s+1)-V(s)) \leq-\delta \sum_{s=n_{0}+\tau}^{n}(|x(s)-p(s)|+|u(s)-q(s)|),
$$

which implies

$$
V(n+1)+\delta \sum_{s=n_{0}+\tau}^{n}(|x(s)-p(s)|+|u(s)-q(s)|) \leq V\left(n_{0}+\tau\right),
$$


that is,

$$
\sum_{s=n_{0}+\tau}^{n}(|x(s)-p(s)|+|u(s)-q(s)|) \leq \frac{V\left(n_{0}+\tau\right)}{\delta} .
$$

It follows from (4.1) that $V_{i}\left(n_{0}+\tau\right)(i=1,2,3)$ are all bounded. Hence

$$
\sum_{s=n_{0}+\tau}^{n}(|x(s)-p(s)|+|u(s)-q(s)|) \leq \frac{V\left(n_{0}+\tau\right)}{\delta}<+\infty
$$

which means that

$$
\sum_{s=n_{0}+\tau}^{+\infty}(|x(s)-p(s)|+|u(s)-q(s)|) \leq \frac{V\left(n_{0}+\tau\right)}{\delta}<+\infty .
$$

This implies that $\lim _{n \rightarrow \infty}(|x(n)-p(n)|+|u(n)-q(n)|)=0$, or $\lim _{n \rightarrow \infty}(x(n)-p(n))=0$, $\lim _{n \rightarrow \infty}(u(n)-q(n))=0$. This completes the proof of Theorem 4.1.

Next, we investigate the existence and uniqueness of an almost periodic sequence solution of system (1.1) by using almost periodic functional hull theory.

Let $\left\{\mu_{k}\right\}$ be any integer valued sequence such that $\mu_{k} \rightarrow \infty$ as $k \rightarrow \infty$. According to Lemma 2.6, taking a subsequence if necessary, we have $\alpha\left(n+\mu_{k}\right) \rightarrow \alpha^{*}(n), \beta\left(n+\mu_{k}\right) \rightarrow$ $\beta^{*}(n), \gamma\left(n+\mu_{k}\right) \rightarrow \gamma^{*}(n), a\left(n+\mu_{k}\right) \rightarrow a^{*}(n), b\left(n+\mu_{k}\right) \rightarrow b^{*}(n), c\left(n+\mu_{k}\right) \rightarrow c^{*}(n)$, as $k \rightarrow \infty$ for $n \in Z$. Then we get a hull equation of system (1.1) as follows:

$$
\begin{aligned}
& \Delta x(n)=-\alpha^{*}(n) x(n)+\beta^{*}(n) x\left(n-\tau_{1}\right) e^{-\gamma^{*}(n) x\left(n-\tau_{1}\right)}-c^{*}(n) x(n) u(n), \\
& \Delta u(n)=-a^{*}(n) u(n)+b^{*}(n) x\left(n-\tau_{2}\right),
\end{aligned}
$$

By the almost periodic theory, we can conclude that if system (1.1) satisfies $\left(H_{1}\right)-\left(H_{4}\right)$, then the hull equation (4.10) of system (1.1) also satisfies $\left(H_{1}\right)-\left(H_{4}\right)$.

From Theorem 3.4 in [29], the following lemma can easily be obtained.

Lemma 4.2 If each hull equation of system (1.1) has a unique strictly positive solution, then the almost periodic difference system (1.1) has a unique strictly positive almost periodic solution.

Theorem 4.3 If the almost periodic difference system (1.1) satisfies $\left(H_{1}\right)-\left(H_{4}\right)$, then the almost periodic difference system (1.1) admits a uniqueness of globally attractive almost periodic sequence solution.

Proof By Lemma 4.2, we only need to prove that each hull equation of system (1.1) has a unique strictly positive solution. We prove that the existence of a strictly positive solution of any hull equations of system (1.1).

By the almost periodicity of $\{\alpha(n)\},\{\beta(n)\},\{\gamma(n)\},\{a(n)\},\{b(n)\}$, and $\{c(n)\}$, there exists an integer valued sequence $\left\{\delta_{k}\right\}$ with $\delta_{k} \rightarrow \infty$ as $k \rightarrow \infty$ such that $\alpha\left(n+\delta_{k}\right) \rightarrow \alpha^{*}(n)$, $\beta\left(n+\delta_{k}\right) \rightarrow \beta^{*}(n), \gamma\left(n+\delta_{k}\right) \rightarrow \gamma^{*}(n), a\left(n+\delta_{k}\right) \rightarrow a^{*}(n), b\left(n+\delta_{k}\right) \rightarrow b^{*}(n), c\left(n+\delta_{k}\right) \rightarrow$ $c^{*}(n)$, as $k \rightarrow \infty$ for $n \in Z$. Suppose that $X(n)=(x(n), u(n))$ is any positive solution of hull 
equation (4.10). Since $\left(H_{1}\right)$ and $\left(H_{2}\right)$ hold, combined with the proof of Theorem 3.1, we have

$$
\begin{aligned}
& m_{1} \leq \liminf _{n \rightarrow \infty} x(n) \leq \limsup _{n \rightarrow \infty} x(n) \leq M_{1}, \\
& m_{2} \leq \liminf _{n \rightarrow \infty} u(n) \leq \limsup _{n \rightarrow \infty} u(n) \leq M_{2} .
\end{aligned}
$$

Therefore

$$
\begin{aligned}
& 0<\inf _{n \in Z^{+}} x(n) \leq \sup _{n \in Z^{+}} x(n)<\infty, \\
& 0<\inf _{n \in Z^{+}} u(n) \leq \sup _{n \in Z^{+}} u(n)<\infty .
\end{aligned}
$$

Let $\varepsilon$ be an arbitrary small positive number. It follows from (4.11) that there exists a positive integer $N_{0}$ such that

$$
m_{1}-\varepsilon \leq x(n) \leq M_{1}+\varepsilon, \quad m_{2}-\varepsilon \leq u(n) \leq M_{2}+\varepsilon, \quad n>N_{0}
$$

Define $x_{k}(n)=x\left(n+\delta_{k}\right)$ and $u_{k}(n)=u\left(n+\delta_{k}\right)$ for all $n \geq N_{0}+\tau-\delta_{k}, k \in Z^{+}$. For any positive integer $q$, it is easy to see that there exist sequences $\left\{x_{k}(n): k \geq q\right\}$ and $\left\{u_{k}(n): k \geq q\right\}$ such that the sequences $\left\{x_{k}(n)\right\}$ and $\left\{u_{k}(n)\right\}$ have subsequences, denoted by $\left\{x_{k}(n)\right\}$ and $\left\{u_{k}(n)\right\}$ again, converging on any finite interval of $Z$ as $k \rightarrow \infty$. Thus we have sequences $\{y(n)\}$ and $\{v(n)\}$ satisfying

$$
x_{k}(n) \rightarrow y(n), \quad u_{k}(n) \rightarrow v(n), \quad \text { for } n \in Z \text { as } k \rightarrow \infty .
$$

This, combined with

$$
\begin{aligned}
\Delta x_{k}(n)= & -\alpha^{*}\left(n+\tau_{k}\right) x_{k}(n)+\beta^{*}\left(n+\tau_{k}\right) x_{k}\left(n-\tau_{1}\right) e^{-\gamma^{*}\left(n+\tau_{k}\right) x_{k}\left(n-\tau_{1}\right)} \\
& -c^{*}\left(n+\tau_{k}\right) x_{k}(n) u_{k}(n) \\
\Delta u_{k}(n)= & -a^{*}\left(n+\tau_{k}\right) u_{k}(n)+b^{*}\left(n+\tau_{k}\right) x_{k}\left(n-\tau_{2}\right)
\end{aligned}
$$

give us

$$
\begin{aligned}
& \Delta y(n)=-\alpha^{*}(n) y(n)+\beta^{*}(n) y\left(n-\tau_{1}\right) e^{-\gamma^{*}(n) y\left(n-\tau_{1}\right)}-c^{*}(n) y(n) v(n), \\
& \Delta v(n)=-a^{*}(n) v(n)+b^{*}(n) y\left(n-\tau_{2}\right) .
\end{aligned}
$$

We can easily see that $(y(n), v(n))$ is a solution of hull equation (4.10) and

$$
m_{1}-\varepsilon \leq y(n) \leq M_{1}+\varepsilon, \quad m_{2}-\varepsilon \leq v(n) \leq M_{2}+\varepsilon, \quad n \in Z .
$$

Since $\varepsilon$ is an arbitrary small positive number, it follows that

$$
m_{1} \leq y(n) \leq M_{1}, \quad m_{2} \leq v(n) \leq M_{2}, \quad n \in Z
$$


that is,

$$
0<\inf _{n \in Z} y(n) \leq \sup _{n \in Z} y(n)<\infty, \quad 0<\inf _{n \in Z} y(n) \leq \sup _{n \in Z} y(n)<\infty
$$

This implies that each hull equation of the almost periodic difference system (1.1) has at least one strictly positive solution.

Now we prove the uniqueness of the strictly positive solution of each hull equation (4.10). Suppose that the hull equation (4.10) has two arbitrary strictly positive solutions $\left(x^{*}(n), u^{*}(n)\right)$ and $\left(p^{*}(n), q^{*}(n)\right)$. Similar to the proof of Theorem 4.1, we define a Lyapunov functional,

$$
V^{*}(n)=V_{1}^{*}(n)+V_{2}^{*}(n)+V_{3}^{*}(n)
$$

where

$$
\begin{aligned}
& V_{1}^{*}(n)=\left|x^{*}(n)-p^{*}(n)\right|+\left|u^{*}(n)-q^{*}(n)\right|, \\
& V_{2}^{*}(n)=\sum_{u=n-\tau_{1}}^{n-1} \frac{1}{e^{2}} \beta\left(u+\tau_{1}\right)\left|x^{*}(u)-p^{*}(u)\right|, \\
& V_{3}^{*}(n)=\sum_{u=n-\tau_{2}}^{n-1} b\left(u+\tau_{2}\right)\left|x^{*}(u)-p^{*}(u)\right| .
\end{aligned}
$$

Calculating the difference of $V^{*}$ along the solution of the hull equation (4.10), similar to the discussion of (4.9), one has

$$
\Delta V^{*}(n) \leq-\delta\left(\left|x^{*}(n)-p^{*}(n)\right|+\left|u^{*}(n)-q^{*}(n)\right|\right), \quad \text { for } n \in Z
$$

We immediately see that $V^{*}(n)$ is a non-increasing function on $Z$. Summing both sides of (4.13) from $n$ to 0 , we have

$$
\delta \sum_{s=n}^{0}\left(\left|x^{*}(s)-p^{*}(s)\right|+\left|u^{*}(s)-q^{*}(s)\right|\right) \leq V^{*}(n)-V^{*}(1), \quad \text { for } n<0 .
$$

Note that $V^{*}(n)$ is bounded. Hence we have $\sum_{s=-\infty}^{0}\left(\left|x^{*}(s)-p^{*}(s)\right|+\left|u^{*}(s)-q^{*}(s)\right|\right)<+\infty$, which implies that

$$
\lim _{n \rightarrow-\infty}\left(x^{*}(n)-p^{*}(n)\right)=0, \quad \lim _{n \rightarrow-\infty}\left(u^{*}(n)-q^{*}(n)\right)=0 .
$$

Let $\varepsilon$ be an arbitrary small positive number. It follows from (4.14) that there exists a positive integer $N_{1}>0$ such that

$$
\left|x^{*}(n)-p^{*}(n)\right|<\frac{\varepsilon}{Q}, \quad\left|u^{*}(n)-q^{*}(n)\right|<\frac{\varepsilon}{Q}, \quad n<-N_{1},
$$

where $Q=2+\frac{\tau_{1} \beta^{+}}{e^{2}}+\tau_{2} b^{+}$. Therefore, for $n<-N_{1}$,

$$
V_{1}^{*}(n) \leq \frac{\varepsilon}{Q}+\frac{\varepsilon}{Q}, \quad V_{2}^{*}(n) \leq \frac{\tau_{1} \beta^{+}}{e^{2}} \frac{\varepsilon}{Q}, \quad V_{3}^{*}(n) \leq \tau_{2} b^{+} \frac{\varepsilon}{Q} .
$$


It follows from (4.12) and the above inequalities that

$$
V^{*}(n) \leq Q \frac{\varepsilon}{Q}=\varepsilon, \quad n<-N_{1},
$$

so $\lim _{n \rightarrow-\infty} V^{*}(n)=0$. Notice that $V^{*}(n)$ is a non-increasing function on $Z$, and then $V^{*}(n) \equiv 0$. That is $x^{*}(n)=p^{*}(n), u^{*}(n)=q^{*}(n)$, for all $n \in Z$. Therefore, each hull equation of system (1.1) has a unique strictly positive solution.

In view of the above discussion, any hull equation of system (1.1) has a unique strictly positive solution. By Theorem 3.1 and Theorem 4.1, the almost periodic system (1.1) has a uniqueness of globally attractive almost periodic solution. The proof is completed.

\section{An example}

The following example shows the feasibility of our main results.

Example 5.1 Consider the following equations:

$$
\begin{aligned}
\Delta x(n)= & -(0.8+0.01 \sin (\sqrt{2} n)) x(n) \\
& +(3.55+0.01 \sin (\sqrt{3} n)) x(n-1) e^{-(0.72+0.01 \sin (n)) x(n-1)} \\
& -(0.1+0.01 \sin (\sqrt{2} n)) x(n) u(n), \\
\Delta u(n)= & -(0.7+0.01 \sin (\sqrt{3} n)) u(n)+(0.1+0.01 \cos (\sqrt{3} n)) x(n-1) .
\end{aligned}
$$

It is easy to calculate that $M_{1} \approx 2.3349, m_{1} \approx 1.7665, M_{2} \approx 0.3722, m_{2} \approx 0.2239$. By simple calculation, we found that conditions $\left(H_{1}\right)-\left(H_{4}\right)$ are satisfied. It follows from Theorem 4.3 that system (5.1) admits uniqueness of a globally attractive almost periodic solution (see Figure 1).

\section{Conclusion}

In this paper, we propose and study the discrete Nicholson's blowflies model with feedback control. In Theorem 3.1, we obtain condition $\left(\mathrm{H}_{2}\right)$ to ensure the permanence of system (1.1), which shows that the feedback control can change the permanence. However, as is well known, the feedback control cannot influence the permanence [10, 21, 22, 24],
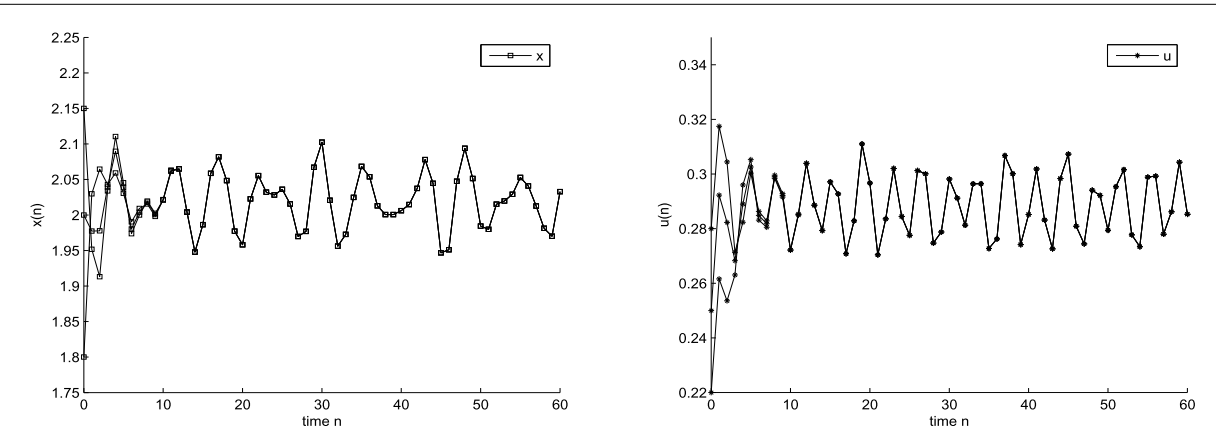

Figure 1 Dynamic behavior of the solution $(x(n), u(n))$ of system (5.1) with the initial conditions $(\varphi(\theta), u(0))=(1.8,0.22),(2,0.25)$, and $(2.15,0.28)$ for $\theta=-2,-1,0$, respectively. 
therefore, the result not only improves but also supplements the known results. Further, by using almost periodic functional hull theory, which is different from that of [6-8], we show that the almost periodic system has a unique strictly positive almost periodic solution, which is globally attractive. The main analysis technique that we use in the proof of the main results is developed from the work of Li et al. [13]. This implies that the main theorems of this paper improve and extend some of previously obtained results.

Competing interests

The authors declare that there is no conflict of interests.

\section{Authors' contributions}

All authors contributed equally to the writing of this paper. All authors read and approved the final manuscript.

\section{Author details}

'Department of Computer Engineering, Fuzhou University Zhicheng College, Fuzhou, Fujian 350002, P.R. China.

${ }^{2}$ Department of Mathematics and Physics, Fujian Institute of Education, Fuzhou, Fujian 350001, P.R. China.

\section{Acknowledgements}

The authors thank the referees for their excellent suggestions that greatly improve the exposition of this paper. Also, the research was supported by the Natural Science Foundation of Fujian Province (2015J01012, 2015J01019).

Received: 19 February 2016 Accepted: 25 May 2016 Published online: 07 July 2016

\section{References}

1. Wang, LJ: Almost periodic solution for Nicholson's blowflies model with patch structure and linear harvesting terms. Appl. Math. Model. 37(4), 2153-2165 (2013)

2. Chen, W, Liu, BW: Positive almost periodic solution for a class of Nicholson's blowflies model with multiple time-varying delays. J. Comput. Appl. Math. 235, 2090-2097 (2011)

3. Fei, L: Positive almost periodic solution for a class of Nicholson's blowflies model with a linear harvesting term. Nonlinear Anal., Real World Appl. 13, 686-693 (2012)

4. Wang, WT: Positive periodic solutions of delayed Nicholson's blowflies models with a nonlinear density-dependent mortality term. Appl. Math. Model. 36, 4708-4713 (2013)

5. Zhang, BG, Xu, HX: A note on the global attractivity of a discrete model of Nicholson's blowflies. Discrete Dyn. Nat. Soc. 3, 51-55 (1999)

6. Alzabut, JO, Bolat, Y, Abdeljawad, T: Almost periodic dynamics of a discrete Nicholson's blowflies model involving a linear harvesting term. Adv. Differ. Equ. 2012, 158 (2012)

7. Alzabut, JO: Existence and exponential convergence of almost periodic positive solution for Nicholson's blowflies discrete model with nonlinear harvesting term. Math. Sci. Lett. 2(3), 201-207 (2013)

8. Yao, ZJ: Existence and exponential convergence of almost periodic positive solution for Nicholson s blowflies discrete model with linear harvesting term. Math. Methods Appl. Sci. 37(16), 2354-2362 (2014)

9. Chen, FD: Permanence of a discrete $\mathrm{N}$-species cooperation system with time delays and feedback controls. Appl. Math. Comput. 186(1), 23-29 (2007)

10. Chen, $L$, Xie, XD, Chen, LJ: Feedback control variables have no influence on the permanence of a discrete $\mathrm{N}$-species cooperation system. Discrete Dyn. Nat. Soc. 2009, Article ID 306425 (2009)

11. Li, Z, Chen, FD, He, MX: Almost periodic solutions of a discrete Lotka-Volterra competition model with delays. Nonlinear Anal., Real World Appl. 12(4), 2344-2355 (2011)

12. Li, Z, Chen, FD: Almost periodic solutions of a discrete almost periodic logistic equation. Math. Comput. Model. 50, 254-259 (2009)

13. Li, Z, Han, MA, Chen, FD: Almost periodic solutions of a discrete almost periodic logistic equation with delay. Appl. Math. Comput. 232, 743-751 (2014)

14. Li, YK, Yang, Y, Wu, WQ: Almost periodic solutions for a class of discrete systems with Allee-effect. Appl. Math. 59(2), 191-203 (2014)

15. Li, YK, Zhang, TW: Permanence and almost periodic sequence solution for a discrete delay logistic equation with feedback control. Nonlinear Anal., Real World Appl. 12(3), 1850-1864 (2011)

16. Chen, FD: Permanence of a discrete $n$-species cooperation system with time delays and feedback controls. Appl. Math. Comput. 186(1), 23-29 (2007)

17. Gopalsamy, K, Weng, PX: Feedback regulation of logistic growth. Int. J. Math. Sci. 16(1), 177-192 (1993)

18. Chen, FD, Liao, XY, Huang, ZK: The dynamic behavior of N-species cooperation system with continuous time delays and feedback controls. Appl. Math. Comput. 181(2), 803-815 (2006)

19. Chen, FD, Yang, JH, Chen, LJ: On a mutualism model with feedback controls. Appl. Math. Comput. 214(2), 581-587 (2009)

20. Chen, FD: Positive periodic solutions of neutral Lotka-Volterra system with feedback control. Appl. Math. Comput. 162(3), 1279-1302 (2005)

21. Chen, $L, X i e, X D$ : Permanence of a $n$-species cooperation system with continuous time delays and feedback controls. Nonlinear Anal., Real World Appl. 12(1), 34-38 (2011)

22. Li, Z, Han, MA, Chen, FD: Influence of feedback controls on an autonomous Lotka-Volterra competitive system with infinite delays. Nonlinear Anal., Real World Appl. 14, 402-413 (2013) 
23. Zhao, $\mathrm{CH}$, Wang, L: Convergence and permanence of a delayed Nicholson's blowflies model with feedback control. J. Appl. Math. Comput. 38, 407-415 (2012)

24. Fan, YH, Wang, LL: Permanence for a discrete model with feedback control and delay. Discrete Dyn. Nat. Soc. 2008, Article ID 945109 (2008)

25. Fink, AM, Seifert, G: Liapunov functions and almost periodic solutions for almost periodic systems. J. Differ. Equ. 5, 307-313 (1969)

26. Hamaya, Y: Existence of an almost periodic solution in a difference equation by Lyapunov functions. Nonlinear Stud. 8(3), 373-379 (2001)

27. Zhang, SN: Existence of almost periodic solution for difference systems. Ann. Differ. Equ. 16(2), 184-206 (2000)

28. Yuan, $R$, Hong, JL: The existence of almost periodic solutions for a class of differential equations with piecewise constant argument. Nonlinear Anal. 28(8), 1439-2450 (1997)

29. Zhang, SN, Zheng, G: Almost periodic solutions of delay difference systems. Appl. Math. Comput. 131, 497-516 (2002)

Submit your manuscript to a SpringerOpen ${ }^{\circ}$ journal and benefit from:

- Convenient online submission

Rigorous peer review

- Immediate publication on acceptance

- Open access: articles freely available online

- High visibility within the field

- Retaining the copyright to your article

Submit your next manuscript at $\boldsymbol{s p r i n g e r o p e n . c o m ~}$ 